\title{
What Happens Beyond Drucker's Proposition in Heterogeneous Media
}

\author{
Yilong Bai ${ }^{1, a}$, Guangwen Ma ${ }^{1, b}$, Shengwang Hao ${ }^{2, c}$, Mengfen Xia ${ }^{1,3, d}$ \\ and Fujiu $\mathrm{Ke}^{1,4, \mathrm{e}}$ \\ ${ }^{1}$ LNM, Institute of Mechanics, Chinese Academy of Sciences, Beijing 100190, China \\ ${ }^{2}$ School of Civil Engineering and Mechanics, Yanshan University, Qinhuangdao 066004, China \\ ${ }^{3}$ Department of Physics, Peking University, Beijing 100871, China \\ ${ }^{4}$ School of Physics and Nuclear Energy Engineering, Beihang University, Beijing 100083, China \\ abaiyl@Inm.imech.ac.cn(corresponding author), ’bmagw@Inm.imech.ac.cn, chsw@ysu.edu.cn, \\ dxiam@Inm.imech.ac.cn, ${ }^{\mathrm{e}} \mathrm{kefj@Inm.imech.ac.cn}$
}

Keywords: continuous bifurcation, damage localization, catastrophic rupture

\begin{abstract}
This paper briefly reviews our recent analytical and experimental results on 3 interrelated features beyond the peak load in heterogeneous media: continuous bifurcation, damage localization and catastrophic rupture (CR). Firstly, an Elastic Statistically-Brittle model (ESB) was introduced to formulate the basic features of a kind of heterogeneous media, like rocks and cements. The global mean field approximation (GMF) shows that the measure of heterogeneity, like the Weibull modulus $\mathrm{m}$ in the distribution of meso-strength plays a key role to distinguish CR from gradual failure. Then, with the ESB model and corresponding experimental results, continuous bifurcation and damage localization are discussed. In accord with these, regional mean field approximation (RMF) is adopted and it shows that any scale of damage localization can satisfy the conservation laws in continuum mechanics. This implies that catastrophic rupture could appear at any state beyond the peak load, depending on the unknown evolution of damage localization zone. Hence, catastrophic rupture seems to occur stochastically at macroscopic level. On the other hand, both experimental and analytic studies demonstrate that a robust power law singularity $(-1 / 2)$ appears ahead of CR. Preliminary applications of these ideas are briefly described.
\end{abstract}

\section{Introduction}

Everyone working in solid mechanics knows Drucker's proposition, beyond which material will become unstable,[1]. But, what may actually happen beyond Drucker's proposition in damaged heterogeneous materials?

In 1965, Cook showed that the failure process of rocks becomes unstable only when the descending slope of the load-displacement curve of a specimen exceeds the slope of the testing machine stiffness, [2]. Then, various types of stiff testing machine were developed. However, it was found that some samples may still break under the high stiff testing machine. Why?

\section{EAB model and critical Weibull modulus}

Now, we start with the ESB model of heterogeneous materials, $[3,4]$. Suppose that the heterogeneous medium consists of linear elastic but brittle mesoscopic units, namely all mesoscopic units have the same elastic modulus E0 but different breaking stress threshold $\sigma \mathrm{c}$, which follows a distribution function. If the distribution follows Weibull distribution, continuum damage mechanics and global mean field (GMF) lead to the following stress-strain relation,

$$
\bar{\sigma}=\bar{\varepsilon} \mathrm{e}^{-\bar{\varepsilon}^{m}}
$$


where the two dimensionless variables are defined as $\bar{\varepsilon}=\frac{E_{0} \varepsilon}{\eta}$ and $\bar{\sigma}=\frac{\sigma}{\eta}$ respectively, $\mathrm{m}$ is Weibull modulus, and $\eta$ is the position parameter in the distribution. Fig. 1 shows the comparison of an experimental observation of a sandstone and the ESB model. One may notice that the smaller the Weibull modulus is, the less the slope of the stress-strain curve is, Fig.1-c.

According to Cook [2], the condition for a stable deformation should be that the increment of the total external work $\Delta \mathrm{W}$ be positive,

$$
\Delta W=\Delta W_{s}+\Delta W_{m}=F \cdot\left(\frac{1}{K_{s}\left(u_{s}\right)}+\frac{1}{K_{m}}\right) \cdot \Delta F>0 .
$$

where $\mathrm{K}$ denotes the stiffness, subscripts $\mathrm{m}$ and $\mathrm{s}$ refer to testing machine and sample respectively. For the ascending part of the force-displacement curve of specimen, i.e. $\mathrm{Ks}>0$ and $\Delta \mathrm{F}>0$, this condition is always satisfied, hence the deformation is always stable. However, for the descending part of the force-displacement curve of specimen, i.e. $\Delta \mathrm{F}<0$, there is a negative sample stiffness $\mathrm{Ks}_{\mathrm{s}}<$ 0 , thus the stable condition can be satisfied only when

$$
K_{m}>-K_{s}\left(u_{s}\right) .
$$

Or say, provided

$$
K_{m} \leq-K_{s}\left(u_{s}\right) .
$$

catastrophic rupture (CR) will appear, see Fig.1-b.

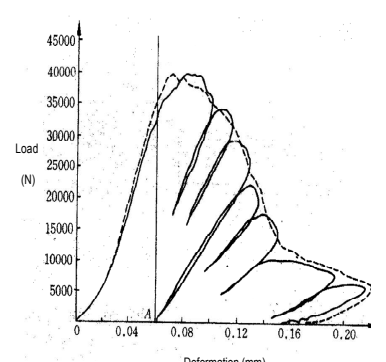

(a)

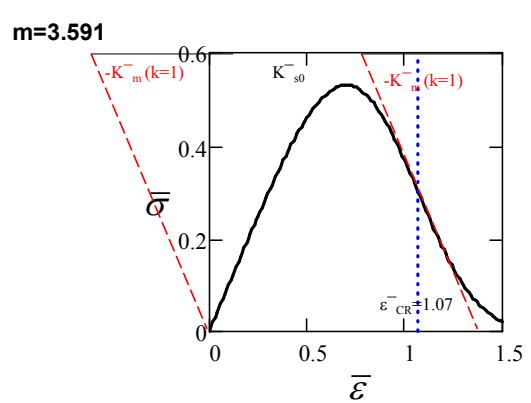

(b)

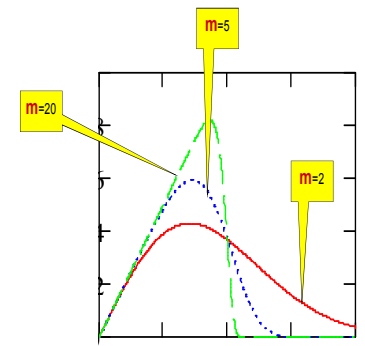

(c)

Fig.1. The curves of load (stress) vs deformation (strain). (a) experimental observation of sandstone (after China Encyclopedia, "Mine and Metallurgy", 1984); (b) ESB model with $\mathrm{m}=3.591$, showing the critical state when $\mathrm{K}_{\mathrm{m}}=\mathrm{K}_{\mathrm{s} 0}$ (.i.e. $\mathrm{k}=1$ ), $\mathrm{K}_{\mathrm{s} 0}$ is the initial stiffness of the sample. (c) ESB model with $\mathrm{m}=20,5$ and 2 .

In particular, if the slope at the inflection point of the load-displacement curve can exactly satisfy $\mathrm{Km}=-\mathrm{Ks}$, it leads to a critical state, as Fig.1-b shows. In the case shown in Fig.1-b, when $\mathrm{m}<$ $\mathrm{mc}=3.591$, there will be no $\mathrm{CR}$ at all and the sample will gradually failure. So, the heterogeneity measure $m$ plays a key role in the appearance of CR.

But, when we compared the strain at CR calculated with GMF, the obtained values are always much greater than those experimentally observed, see Table 1. Why?

\section{Continuous bifurcation and damage localization}

As a matter of fact, continuous bifurcation can appear beyond the strain at peak load, Fig.2. Thus, damage would become localized [5,6], as shown in Fig.3. 


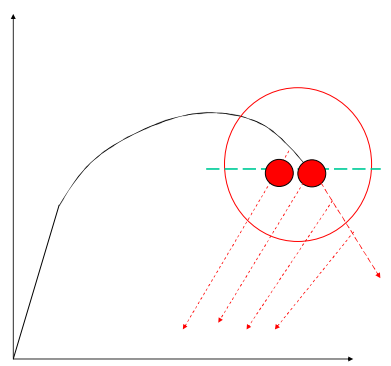

Fig.2 Continuous bifurcation

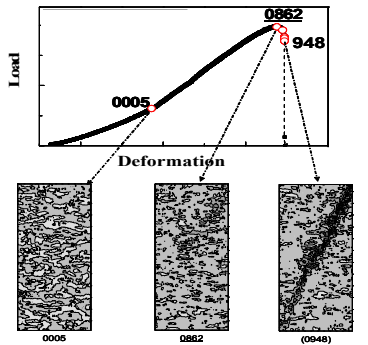

Fig.3 Localization of deformation

(Courtesy of $\mathrm{Xu} \mathrm{XH)}$

As the ESB model shows, with increasing deformation in this phase, a damaged sample could be split into two parts in series: a continuing damage zone and a zone with elastic unloading. The damage zone can be continuously split into such two parts, see Fig.4-a. Accordingly, the nominal strain of the sample would depend on the accumulated outcome of damage localization $\gamma$ as shown in the integral,

$$
\bar{\varepsilon}_{n o \min a l}(\bar{\sigma})=\frac{\bar{\sigma}}{\bar{E}_{u}\left(\bar{\sigma}_{M}\right)}-\bar{\sigma} \int_{\bar{\sigma}_{M}}^{\bar{\sigma}} \gamma\left(\bar{\sigma}_{u}\right) \frac{\bar{E}_{u}{ }^{\prime}\left(\bar{\sigma}_{u}\right)}{\bar{E}_{u}^{2}\left(\bar{\sigma}_{u}\right)} \cdot d \bar{\sigma}_{u} .
$$

where Eu denotes elastic unloading modulus and subscript $\mathrm{u}$ denotes the starting point of unloading. Clearly, the evolution of localization zone is a key player in the process beyond peak load, [4]. Fig.4-b shows some of the evolution of the localized zone experimentally observed.

\section{Catastrophic rupture and its $-1 / 2$ power law singularity}

Since catastrophic rupture should be a self-sustainable process in energy aspect, under stiff loading, the energy dissipated in the process of catastrophe should be supplied by the energy released from the elastically unloading zone of the sample, regardless of external work. Thus, the condition for CR would become

$$
\frac{\bar{\sigma} \bar{E}_{u}^{\prime}(\bar{\sigma})}{\bar{E}_{u}^{2}(\bar{\sigma})} \gamma(\bar{\sigma})-\int_{\bar{\sigma}}^{\bar{\sigma}_{M}} \frac{\bar{E}_{u}^{\prime}\left(\bar{\sigma}_{u}\right)}{\bar{E}_{u}^{2}\left(\bar{\sigma}_{u}\right)} \gamma\left(\bar{\sigma}_{u}\right) d \bar{\sigma}_{u}^{>} \leq \frac{1}{\bar{E}_{u}\left(\bar{\sigma}_{M}\right)}, \quad \begin{gathered}
\text { Stable } \\
C R
\end{gathered}
$$

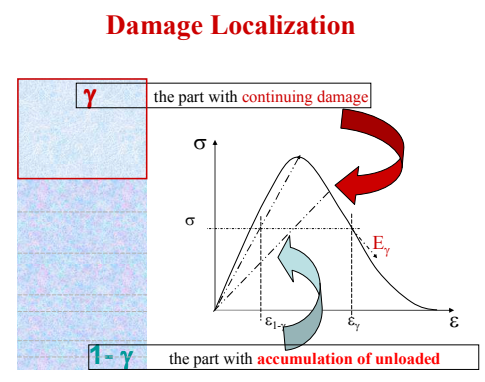

(a)

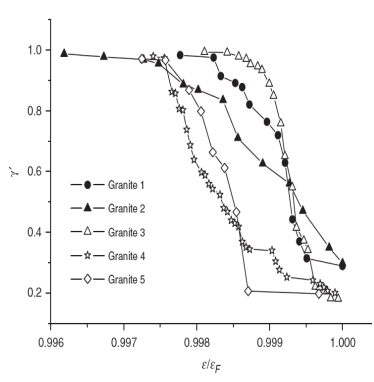

(b)

Fig.4 Damage localization. (a) A sketch of localization and its relation to continuous bifurcation. (b) Experimentally measured localization of granite under compression, (after Hao, et al [4])

As the size of the damage localization zone becomes smaller, catastrophic rupture becomes more likely to appear, [4].

Unfortunately, the analysis of continuum damage mechanics shows that any scale of damage localization $\gamma$ can satisfy the conservation laws in continuum mechanics. This implies that a catastrophic rupture could appear at any moment beyond peak load with a specific size of damage localization zone. The narrower the damage zone is, the nearer to the peak point the catastrophic rupture appears. In this sense, catastrophic rupture could not be forecasted in terms of continuum 
damage mechanics. Table 1 gives a comparison of the strains at CR in terms of various approaches: experimental measurement, GMF and RMF approximations. It shows that the calculated values based on combined RMF and experimentally measured width of localized zone are in good agreements with experimental results with error $\pm 6 \%$, [6,7].

Table 1 Comparison of the strains when catastrophic rupture occurs, experimentally measured and calculated values with GMF and RMF, with the experimentally observed width of localized zone, (after Hao [7]).

\begin{tabular}{|c|c|c|c|}
\hline Exp strain at CR & $\begin{array}{c}\text { Cal strain at CR } \\
(\text { RMF with } \gamma)\end{array}$ & $\begin{array}{c}\text { Cal strain at CR } \\
\text { (GMF) }\end{array}$ & $\begin{array}{c}\text { Width of } \\
\text { localized zone } \gamma \\
\text { (Exp) [mm] }\end{array}$ \\
\hline 0.00850 & 0.00816 & 0.00879 & 7.1 \\
\hline 0.00655 & 0.00694 & 0.00839 & 7.9 \\
\hline 0.00645 & 0.00674 & 0.00810 & 6.7 \\
\hline 0.00636 & 0.00611 & 0.00694 & 7.9 \\
\hline 0.00634 & 0.00633 & 0.00682 & 6.3 \\
\hline 0.00515 & 0.00606 & 0.00661 & 6.9 \\
\hline error & $\pm 6 \%$ & $\sim+30 \%$ & \\
\hline
\end{tabular}
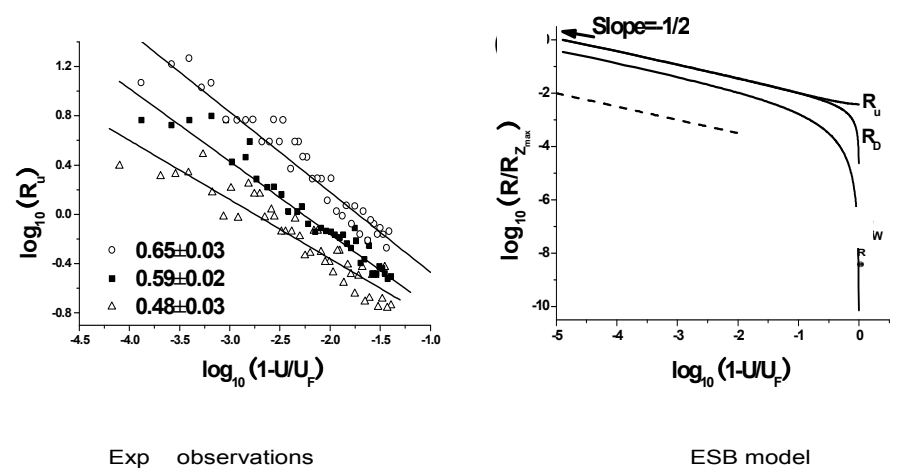

Fig. 5 The $-1 / 2$ power law singularity ahead of Catastrophic rupture shown in log-log plot, R denotes the response and $U$ is the boundary displacement, subscript $F$ denotes the state of $C R$, Hence, the extreme left hand of the abscissa tends to the CR point. (a) Experimental results; (b) ESB model, (after Hao [7]).

As a matter of fact, the uncertainty of such catastrophic rupture is rooted in the complexity of the combination of the distributions of mesoscopic structures, stress-redistribution and damage evolution, etc. Hence, to predict the critical state of catastrophic rupture is far beyond the knowledge of available continuum damage mechanics.

However, in practice, from the experimental and analytic studies we found that at least there are two possibilities to foresee catastrophic rupture (CR). The first is to monitor the evolution of damage localization as mentioned above. The second is to check if a $-1 / 2$ power law singularity appears or not. Both our experimental results and theoretical analysis show that the responses of a heterogeneous sample to external variable will present a $-1 / 2$ power law singularity ahead of CR, as shown in Fig.5, whereas gradual failure does not demonstrate such singularity at all. This is a common critical singularity of CR and may serve as a precursor to CR. 


\section{Preliminary applications}

The applications of these ideas to M9 and M8 earthquakes in Japan and Wenchuan, are quite encouraging.

Based on the GPS observations of M9 Tohoku Earthquake, the distribution of co-seismic incremental strains are reconstructed and then the elastic energy released from an unloading region is calculated as about $8 \times 1018 \mathrm{~J}$, when the critical strain increment is taken to be $1 \times 10-6$, where energy release almost saturated. The value of energy release is in reasonable agreement with the energy involved in seismic waves. In addition, the unloading strain increments $1 \times 10-6$ leads to a relevant area of $\sim 2 \times 105 \mathrm{~km} 2$ (roughly $400 \mathrm{~km} \times 500 \mathrm{~km}$ to the northwest of the faults), [8].

Based on the GPS observations before M8 Wenchuan Earthquake, The reconstructed strain distributions show that the zone with localized deformation, about $-105 \mathrm{~km}<\mathrm{Y}<25 \mathrm{~km}$, covers the earthquake faults $(\mathrm{Y}=0)$. In particular, the reconstructed responses based on the last two recordings before the quake demonstrate nearly $-1 / 2$ power law singularity, [9]. Of course, further data analysis is still needed to justify the results.

\section{Summary}

Beyond the strain at peak load, owing to continuous bifurcation, damage localization appears in a sample of heterogeneous material. In accord with Elastic Statistically-Brittle (ESB) model, the damage localization is formulated in terms of continuum mechanics. It is shown that catastrophic rupture could not be forecasted in the framework of continuum mechanics, if the evolution of localization is unknown. However, both experimental and analytic studies indicate that $-1 / 2$ power law singularity could serve as precursors to foresee catastrophic rupture.

\section{Acknowledgements}

This work is supported by the National Natural Science Foundation of China (Grant Nos. 11021262 and 10972218), the National Basic Research Program of China (973 Program), and the CAS Innovation Program.

The contributions made by Drs Xu XH, Rong F and Lu MF are greatly appreciated.

\section{References}

[1] D.C. Drucker, Introduction to the Mechanics of Deformable Solids, McGraw-Hill, New York, 1967.

[2] N.G.W. Cook, The failure of rock, Int. J. Rock Mech. Min. Sci. 2 (1965) 389-403.

[3] D. Krajcinovic, Damage Mechanics, North-Holland, Amsterdam, 1996.

[4] S.W. Hao, M.F. Xia, F.J. Ke and Y.L. Bai, Evolution of Localized Damage Zone in Heterogeneous Media, Int. J. Damage Mechanics. 19 (2010) 787-804.

[5] X.H. Xu, S.P. Ma, M.F. Xia, F.J. Ke and Y.L. Bai, Damage evaluation and damage localization of rock, Theo. Applied Fract. Mech.. 42(2) (2004) 131-138.

[6] S.W. Hao, H.Y. Wang, M.F. Xia, F.J. Ke and Y.L. Bai, Relationship between strain localization and catastrophic rupture, Theo. Applied Fract. Mech.. 48 (2007) 41-49.

[7] S.W. Hao, Damage Localization, Catastrophic Rupture and Critical Singularity in Heterogeneous Media under Biaxial Loading, PhD thesis, Institute of Mechanics, CAS, 2007.

[8] M.F. Lu, L. Li, M.F. Xia, H.Y. Wang, Y.L. Bai, Co-seismic energy release and relevant region of Tohoku M9.0earthquake, Sci. China Earth Sci.. 54 (2011) 947-950.

[9] M.F. Lu, Damage induced Catastrophic Rupture in Heterogeneous Media under Biaxial Loading, PhD thesis, Institute of Mechanics, CAS, 2011. 


\section{Advances in Engineering Plasticity XI}

10.4028/www.scientific.net/KEM.535-536

\section{What Happens beyond Drucker's Proposition in Heterogeneous Media}

10.4028/www.scientific.net/KEM.535-536.3

\section{DOI References}

[2] N.G.W. Cook, The failure of rock, Int. J. Rock Mech. Min. Sci. 2 (1965) 389-403.

http://dx.doi.org/10.1016/0148-9062(65)90004-5

[4] S.W. Hao, M.F. Xia, F.J. Ke and Y.L. Bai, Evolution of Localized Damage Zone in Heterogeneous Media, Int. J. Damage Mechanics. 19 (2010) 787-804.

http://dx.doi.org/10.1177/1056789509359660

[6] S.W. Hao, H.Y. Wang, M.F. Xia, F.J. Ke and Y.L. Bai, Relationship between strain localization and catastrophic rupture, Theo. Applied Fract. Mech. 48 (2007) 41-49.

http://dx.doi.org/10.1016/j.tafmec.2007.04.006

[8] M.F. Lu, L. Li, M.F. Xia, H.Y. Wang, Y.L. Bai, Co-seismic energy release and relevant region of Tohoku M9. 0earthquake, Sci. China Earth Sci. 54 (2011) 947-950.

http://dx.doi.org/10.1007/s11430-011-4229-7 